\title{
TOPOLOGICALLY TRIVIAL DEFORMATIONS OF ISOLATED QUASIHOMOGENEOUS HYPERSURFACE SINGULARITIES ARE EQUIMULTIPLE
}

\author{
DONAL B. O'SHEA
}

\begin{abstract}
It is shown that any topologically trivial (and, hence, any $\mu$-constant) deformation of an isolated quasihomogeneous hypersurface singularity is equimultiple.
\end{abstract}

Introduction. In his retiring Presidential address to the American Mathematical Society in 1971, Zariski asked whether two hypersurface singularities which were homeomorphic as embedded varieties necessarily had the same multiplicity [10]. Despite Zariski's allowance that he would be "greatly disappointed" if topologists did not settle the issue in short order, his question remains unanswered.

Even special cases of Zariski's problem have proved intractable. For instance, a necessary and sufficient condition that the hypersurfaces in a family of isolated hypersurface singularities all be homeomorphic as embedded varieties is that family be $\mu$-constant (for necessity, see, for example, [7]; for sufficiency, see [5], when the dimension is not two; see [6], for an announcement in the two-dimensional case). Thus, one might ask whether $\mu$-constant families of singularities are equimultiple. This is known to be the case if the family satisfies the Whitney condition [4] (or, equivalently, [8], if the family is $\mu^{*}$-constant). However, not all $\mu$-constant families are $\mu^{*}$-constant [2].

The purpose of this note is to show that a particular class of $\mu$-constant families of singularities are equimultiple. This class includes all known examples of $\mu$-constant families which are not $\mu^{*}$-constant. More specifically, we prove the following.

THEOREM. Any family of hypersurface singularities which is a $\mu$-constant deformation of an isolated quasihomogeneous singularity is equimultiple.

I am very grateful to D. von Straten, who called my attention to Varchenko's result (Proposition 2 below). The original version of this paper was much longer, most of it being devoted to the proof of a weaker version of Proposition 2.

Received by the editors November 15, 1985 and, in revised form, July 7, 1986.

1980 Mathematics Subject Classification (1985 Revision). Primary 32G11, 14B07, 32C40.

This work was supported in part by the National Science Foundation. 
Proof of the theorem. We fix a coordinate system $x_{1}, \ldots, x_{n}$ on $\mathbf{C}^{n}$.

Definition. Let $\mathbf{w}=\left(w_{1}, \ldots, w_{n}\right)$ be a fixed vector in $\left(\mathbf{R}^{+}\right)^{n}$. We say that a monomial $x_{1}^{a_{1}} \cdots x_{n}^{a_{n}}$ has $\mathbf{w}$-degree $d$ if $\sum w_{i} a_{i}=d$. Suppose that $f: \mathbf{C}^{n}, \mathbf{0} \rightarrow \mathbf{C}, 0$ is a quasihomogeneous polynomial with weights $\mathbf{w}$ and $\mathbf{w}$-degree $d$ (that is, suppose that $f$ can be written as a linear combination of monomials of w-degree $d$ ). A deformation $F: \mathbf{C}^{n} \times \mathbf{C}, \mathbf{0} \times \mathbf{C} \rightarrow \mathbf{C}, 0$ of $f$ will be said to be upper if the expansion

$$
F(\mathbf{x}, t)=f(\mathbf{x})+t f_{1}(\mathbf{x})+t^{2} f_{2}(\mathbf{x})+\cdots
$$

of $F$ in powers of the deformation parameter $t$ is such that each $f_{i}(\mathbf{x})$ is a linear combination of monomials of $\mathbf{w}$-degree greater than or equal to $d$. (The term upper refers to the fact that the deformation involves adding terms on or above the Newton polygon of $f$.) As usual, for fixed $t \in \mathbf{C}$, we let $F_{t}: \mathbf{C}^{n}, \mathbf{0} \rightarrow \mathbf{C}, 0$ be the function defined by setting $F_{t}(\mathbf{x})=F(\mathbf{x}, t)$. Let $m_{t}$ denote the multiplicity and $\mu_{t}$ the Milnor number (if defined) of $F_{t}$ at the origin. The deformation $F$ is equimultiple (resp., $\mu$-constant) if $m_{0}=m_{t}$ (resp., $\mu_{0}=\mu_{t}$ ) for all $t$ sufficiently close to 0 .

Proposition 1. If $f$ is a quasihomogeneous polynomial with an isolated singularity at the origin, then any upper deformation of $f$ is equimultiple.

Proof. Let $\mathbf{x}=\left(x_{1}, \ldots, x_{n}\right)$ be variables and $\mathbf{w}$ be weights with respect to which $f$ is quasihomogeneous. Let $d$ be the w-degree of $f$. Because $f$ has an isolated singularity, for each $i, 1 \leqslant i \leqslant n$, we can choose $j_{i}$ to be some (say, the least) $k$, $1 \leqslant k \leqslant n$, for which the monomial $x_{i}^{b_{i}} x_{k}$ appears in the expansion of $f$ as a linear combination of monomials. (Here, we allow $i=k$. If $j_{i}$ were not to exist for some $i$, then the entire $x_{i}$-axis would consist of singular points-an observation due to Arnol'd [1].) By renumbering, we may assume that $b_{1} \leqslant b_{2} \leqslant \cdots \leqslant b_{n}$.

Let $\mathbf{x}^{a}=x_{1}^{a_{1}} \cdots x_{n}^{a_{n}}$ be a monomial with $\mathbf{w}$-degree greater than or equal to $d$. I claim that the multiplicity of $\mathbf{x}^{a}$ is greater than $b_{1}$. This will, at one stroke, show that the multiplicity of $f$ is $b_{1}+1$, and establish the theorem.

To establish the claim, suppose first that the $\mathbf{w}$-degree of $\mathbf{x}^{a}$ is equal to $d$. For each $i, 1 \leqslant i \leqslant n$, we have

$$
b_{i} w_{i}+w_{j_{i}}=d
$$

Multiply the $i$ th equation by $a_{i}$, and write $b_{i}=b_{1}+c_{i}$ where $c_{i} \geqslant 0$. Sum over all $i$, and transpose to get

$$
\left(b_{1}-\sum a_{i}\right) d+\sum a_{i} c_{i} w_{i}+\sum a_{i} w_{j_{i}}=0 .
$$

Since the third term is strictly positive and the second nonnegative, the above relation cannot hold unless the multiplicity $\sum a_{i}$ of the monomial $\mathbf{x}^{a}$ is strictly greater than $b_{1}$. A trivial modification of the above argument takes care of the case of higher w-degree and completes the proof.

This establishes the theorem in what appears to be a special case. However, we now invoke the result following, which is an immediate corollary of a theorem due to Varchenko [9] (see also [2, Theorem 8 of Chapter III.14, p. 292]). 
Proposition 2. Any $\mu$-constant deformation of a quasihomogeneous polynomial with an isolated singularity is upper.

Varchenko's proof of the above result uses mixed Hodge theory to estimate the codimension of the $\mu$-constant stratum in a versal deformation of a quasihomogeneous polynomial. We remark that the converse of Proposition 2 is also true, and is considerably easier to prove.

Note. Gert-Martin Greuel (Univ. Kaiserslautern) has independently proved the main theorem of this paper. His proof also uses Varchenko's theorem, but is otherwise quite different from ours.

\section{REFERENCES}

1. V. I. Arnol'd, Normal forms of functions in the neighbourhood of degenerate critical points, Russian Math. Surveys 29 (1974), 19-48.

2. V. I. Arnol'd, S. M. Gusein-Zade, and A. N. Varchenko, Singularities of differentiable mappings. The monodromy and asymptotic integrals, Nauka, Moscow, 1984. (Russian)

3. J. Briancon and J.-P. Speder, La trivialité topologique n'implique pas les conditions de Whitney, C. R. Acad. Sci. Paris 280 (1975), 365-367.

4. H. Hironaka, Normal cones in analytic Whitney stratifications, Inst. Hautes Études Sci. Publ. Math. 36 (1969), 127-138.

5. Le Dung Trang and C. P. Ramanujam, The invariance of Milnor's number implies the invariance of the topological type, Amer. J. Math. 98 (1976), 67-78.

6. B. Perron, " $\mu$ constant" implique "type topologique constant" en dimension complexe trois, $\mathrm{C} . \mathrm{R}$. Acad. Sci. Paris 295 (1982), 735-738.

7. B. Teissier, Deformations a type topologique constant. II, Seminaire Douady-Verdier 1972, Secretariat E. N. S. Paris, 45 rue d'Ulm.

8. Cycles evanescents. sections planes et conditions de Whitney, Asterisque 7-8 (1973), 285-362.

9. A. N. Varchenko, $A$ lower bound for the codimension of the stratum $\mu=$ const in terms of the mixed Hodge structure, Vestnik Moskov. Univ. Ser. I Mat. Mekh. 37 (1982), 28-31. (Russian)

10. O. Zariski, Some open questions in the theory of singularities, Bull. Amer. Math. Soc. 77 (1971), 481-491.

Department of Majhematics, Mount Holyoke College, South Hadley, Massachusetts 01075 